\title{
Sleep, Adenosine and Caffeine as Tools for The Early Diagnosis of Parkinson Disease
}

\author{
Rafael J. Salin-Pascual ${ }^{*}$ \\ Department of Psychiatry and Mental Health. School of Medicine. National Autonomous University of Mexico
}

\begin{abstract}
The information that has developed regarding Parkinson's Disease (PD) allowed to understand that there is a period of this disease, perhaps at least a decade, in which the classical features of PD are not detected as such, however, there are evidence of a series of non-motor manifestations, among which are: anosmia, constipation, psychiatric disorders such as major depression, generalized anxiety, and rapid eye movement sleep behavior disorder (RBD).

On the other hand the understanding of physiological mechanisms of sleep-wake cycle, have become remarkable tools to explore neurological and psychiatric disorders and complemented the information and treatments for those disorders.

There is evidence that RBD can occur in half of people who will develop PD, about 10 year letter. The role of adenosine in sleep and caffeine intolerance is other aspects that are evident in the relation between PD and sleep. The detection of PD in early stages, may allow the implementation of the type of therapeutic strategies for neuroprotection. The general goal would be delay or reduce the clinical manifestations of PD in some patients.
\end{abstract}

Keywords: Parkinson Disease, RBD, Adenosine, Caffeine, Sleep.

\section{INTRODUCTION}

Sleep could be seen as a complex behavior that in humans could give us some information about physiological process as circadian rhythms, neuroendocrine system, body energy regulation and at the same time, by studying sleep in pathological process, give us some light into the physiopathology of such diseases. In the last half-century sleep research both basic and clinical, has increased our knowledge about mechanism and functions of such behavior. Because humans spend about one-third of each day in sleep, that could be a powerful tool for to understand morbid process [1-3]

The central goal of this review is to present some findings about the relationship between Parkinson's disease, sleep mechanism, and adenosine as sleep factor, its receptors, the prevalence of some sleep disorders in that neurodegenerative affection, and to figure out if the hypersensitivity to caffeine, could be a cue for to organize some forms of PD with premorbid sleep disorders as well as anxiety problems. Those connections could be useful in to employ some strategies for identifying premorbid cases, that will help us to implement approaches as neuroprotection that could slow down the natural course of that disease.

\section{PARKINSON DISEASE}

Parkinson Disease (PD) is an incurable progressive neurological condition caused by a degeneration of

*Address correspondence to this author at the Department of Psychiatry, School of Medicine, National Autonomous University of Mexico, Hidalgo 187 casa 7, Barrio del Niño Jesus - Tlalpan, Mexico City 14080, Mexico; Tel: 52-55734198; E-mail: rafasalin@yahoo.com dopamine-producing cells at the substantia nigra (SN). The most common symptoms of that condition include slowness of movement, tremor and muscle stiffness; other symptoms include sleep difficulties, depression and anxiety. Parkinson's is usually controlled by medication but other treatments and therapies, such as occupational therapy, physiotherapy and speech and language therapy, can also be effective in controlling and managing symptoms. [4]. Recently Deep Brain Stimulation (DBS) have been developed as an important therapeutic strategy for help some PD patients. Those with intractable motor fluctuations, intractable tremor, or intolerance of medication, and adverse effects are good candidates for DBS [5-7].

Initial estimates based on nigra neuropathological findings or striatal dopamine imaging suggested a five to six year of preclinical period. Lewy body pathology in other neuronal populations preceding nigra involvement suggests that the preclinical phase may be much longer [8]. Epidemiologic studies of non-motor manifestations, such as constipation, anxiety disorders, and rapid eye movement sleep behavior disorder (RBD), and anemia; suggest that the preclinical period extends at least 20 years before the motor manifestations [9-11]. Olfactory impairment and depression are also two problems that anticipate motor manifestations [12].

The pathological process in the nervous system that will lead to PD begins decades before the typical motor symptoms and by the time the diagnosis is made, about $70 \%$ to $80 \%$ of striatal dopamine (DA) and at least one-third of SN neurons and striatal dopaminergic fibers are already lost [13].

$\mathrm{PD}$ is a multisystem disorder though its more relevant symptoms derive from a disruption of the physiology of cortical-basal ganglia-thalamic-cortical circuits $[14,15]$. 
These circuits function as a highly ordered and interconnected network with multiple feedback regulatory mechanisms. There are several sub circuits with intrinsic neurophysiological properties and regulatory systems [15]. Compensation can be illustrated by the well-known fact that the majority of acute and focal lesions of basal ganglia are unnoticed by the patient until the affected structures are beyond compensation. Moreover, when they are symptomatic (i.e. hemiballismus by a sub-thalamic lesion), a relatively rapid recovery occurs. Thus, these structures are extremely plastic. Plasticity refers to the ability of the nervous system to adapt to new situations by changing the effectiveness of transmission in neural circuits [16, 17]. It is almost always regarded as a useful phenomenon that is important in learning and memory and could potentially compensate for dysfunction after injury.

Several characteristics of PD are clear expressions of compensatory mechanisms. Firstly, pathological studies have showed that a proportion as high as $30 \%$ of normal individuals has alpha-synuclein inclusions in their brains and that $15 \%$ have Lewy bodies. Secondly, PD has a long presymptomatic period of at least 5-6 years. This suggests that many neurons in the SNpc can die before they are missed at the clinical level. Parkinsonian signs appear when dopaminergic neuronal death exceeds a critical threshold (70-80\% of striatal nerve terminals and $50-60 \%$ of SN cell bodies). Thirdly, its progression is slow and gradual. Finally, there is a non-linear relationship between dopaminecontaining neuron loss and functional impairment [18]. These points result largely from adaptive neurochemical changes that occur within the striatum or in other parts of the basal ganglia circuits [18].

Some of the premorbid symptoms of PD (also known as non-motor symptoms) are the following:

Anosmia: Olfactory dysfunction is an appealing biomarker for PD because of the high prevalence $(>90 \%)$ among PD patients and the relative ease of testing, as compared to other putative biomarkers such as neuroimaging of the dopamine system. Hyposmia develops during the early stages of PD and, therefore, may be one of the most sensitive markers for the early diagnosis of PD [19].

Constipation: Symptoms of dysautonomia develop in most patients during the course of $\mathrm{PD}$, and constipation is probably the most common manifestation. Constipation relates to impaired colonic mobility and is not simply attributable to medications. Previous authors have commented that constipation may sometimes precede the initial motor manifestations of PD. This finding was borne out in the Honolulu-Asia Aging Study, in which men without PD or dementia were prospectively followed up after completing a bowel-movement questionnaire. Incidental Lewy body pathology was present in nearly one-fourth of individuals with constipation (one bowel movement daily) compared with $6.5 \%$ of individuals without it [20].

Anxiety and other psychiatric disorders: Several casecontrol or cohort studies suggested that anxiety may be one of the earliest manifestations of PD. First, in a populationbased, case-control studies, anxiety diagnoses documented in historical medical records were significantly associated with later PD, [21].
Several investigators have also commented on premorbid parkinsonian personality. Twin and other studies have suggested that patients with PD, even since childhood, are more introverted, serious, cautious, tense, nervous, obsessive-compulsive, inflexible, industrious, and honest; that they avoid risk-seeking behavior such as smoking; and that they are less likely to exhibit impulsive or noveltyseeking behavior compared with controls. Despite these characteristics psychological and personality changes occurring even in early stages of PD, they are relatively nonspecific, and it is unlikely that these traits can be used to reliably predict increased risk of $\mathrm{PD}$ in the general population [22]. Sleep problems are part of the non-motor symtoms.

\section{SLEEP DISORDERS AND PD}

PD patients often experience sleep symptoms like insomnia, parasomnias, and daytime somnolence early on and when motor manifestations are observed. Daytime sleepiness, restless leg syndrome, periodic limb movements disorder and REM sleep behavior disorders are the more prevalent. The last one was figured out as one of the early manifestations of PD even before any motor symptoms. $\mathrm{RBD}$ is characterized by vigorous movements during REM sleep, and may also be caused by neuronal degeneration in the central nervous system (CNS) [23]. There are also some cases of late-onset sleep walking [24].

There are some proposal that about at least $50 \%$ of patients with RBD could develop PD, and it was hypothesized that many of these potential early PD markers (anosmia, autonomic symptoms, psychiatry and soft neurological signs plus cognitive decline), would be also early present in patients with idiopathic $\operatorname{RBD}[25,26]$. In 2006 , it was reported, as the results of a small case control study that documented substantial abnormalities of olfaction, color vision, autonomic function and motor function in patients with idiopathic RBD [27].

There were evaluated an array of potential PD predictive markers in 159 subjects; including 68 with idiopathic RBD, 36 controls, 34 PD patients with RBD and 21 PD patients without RBD. Compared with controls, patients with idiopathic RBD demonstrated substantial olfactory loss. Olfaction was more impaired in PD than idiopathic RBD and did not differ between PD patients with, or without, RBD. Numerous measures of motor function including the Unified Parkinson Disease Rating Scale, alternate tap. Purdue Peg Board and Timed "Up and Go", were impaired in idiopathic RBD compared with controls. All of these motor measures were worse in PD than with idiopathic RBD, regardless of RBD status. Autonomic symptoms and systolic blood pressure drop was impaired in patients with idiopathic RBD compared with controls. Orthostatic abnormalities in PD were found in the group with RBD. However, Parkinson's patients without RBD were not different than controls and had less impairment than those with idiopathic RBD and PD patients with RBD. This study could give an idea that RBD is a premorbid condition that could evolve to $\mathrm{PD}$. There are some recent research and reviews that support that [28-30].

There are some different approaches in order to clarify the relationship between sleep and PD. Does sleep duration could be related to PD? What are the relationship between 
long sleepers and short sleepers and the incidence of PD? Two groups of nurses were studied. The study follows a working rotating night shift, and it was looking for the association with the risk of PD among 84,794 female nurses who reported years of night shift work in 1988 (the US Nurses' Health Study). After 975,912 person-years of followup (1988-2000), 181 incident PD cases were documented. Compared with nurses who never worked rotating night shifts, those with 15 years or more of night shift work had a $50 \%$ lower risk of PD after adjustment for age and smoking. Sleep duration was positively associated with PD risk: The relative risk was 1.84 (95\% confidence interval: $0.99,3.42$ ) when comparing nurses who reported 9 or more hours of sleep per day with those who slept 6 hours or less ( $\mathrm{p}$ trend $=$ 0.005). These data suggest that working night shifts may be protective against PD or that low tolerance for night shift work is an early marker of PD [31].

Another approach for to understand the relationship between sleep and PD is looking into the phenomenology of RBD. Are PD patients showing some motor problems when they are acting their dreams content?

In the following study [32], it was interviewed one hundred consecutive non-demented patients with PD and their bed partners using a structured questionnaire assessing the presence of RBD. They rated the quality of movements, voice and facial expression during $\mathrm{RBD}$ as being better, equal or worse than in awake as in the "ON levodopa condition". Nighttime sleep and movements were videomonitored during polysomnography in 51 patients to evaluate the presence of bradykinesia, tremor and hypophonia during RBD. Fifty-nine patients had clinical RBD with $53 / 59$ bed partners that were able to evaluate them. All $53(100 \%)$ reported an improvement of at least one component of motor control during RBD. By history, movements were improved in $87 \%$ patients (faster, $87 \%$; stronger, $87 \%$; smoother, $51 \%$ ), speech was better in $77 \%$ patients (more intelligible, 77\%; louder, 38\%; better articulated, 57\%) and facial expression was normalized in $47 \%$ patients. Thirty-eight per cent of bed partners reported that movements were 'much better', even in the most disabled patients. Video observations purposeful of motor activity at the moment of REM sleep demonstrate that no rigidity was observed, also surprisingly fast, ample, coordinated and symmetrical motor activity, without obvious sign of Parkinsonism. The movements were, however, jerks, violent and often repetitive. While all patients had asymmetrical parkinsonism when awake, most of the time they used the more disabled arm, hand and leg during the RBD $(P=0.04)$. Movements involved six times as often the upper limbs and the face as the lower limbs (OR: 5.9, P = 0.004). The percentage of time containing tremor EMG activity decreased with sleep stages from 34.9 to $15.5 \%$ during wakefulness, to 3.6 to $5.7 \%$ during non-REM sleep stages $1-2,1.463 .0 \%$ during non-REM sleep stages 3-4, and $0.0660 .2 \%$ during REM sleep (in this last case, it was subclinical tremor) [32].

The restored motor control during REM sleep suggests a transient 'levodopa-like' reestablishment of the basal ganglia loop. Alternatively, Parkinsonism may disappear by REM sleep-related disjunction between pyramidal and extrapyramidal systems. The researcher in this study suggests the following model: the movements during the RBD would be generated by the motor cortex and would follow the pyramidal tract bypassing the extrapyramidal system.

These movements would eventually be transmitted to lower motor neurons because of brain stem lesions interrupting the ponto-medullary pathways which mediate the REM sleep atonia [32].

Hypersomnia or daytime sleepiness is also a premorbid manifestation in PD. There are also some narcoleptic-like symptoms. PD is preceded and accompanied by daytime sleep attacks, nocturnal insomnia, REM sleep behavior disorder, hallucinations and depression, symptoms that are frequently as troublesome as the motor symptoms. Some of these symptoms are present also in narcolepsy, which is linked to a selective loss of hypocretin (Hcrt) neurons. With a sample of brain slides from PD patients and normal controls, it was found an increasing loss of hypocretin cells with disease progression [33, 34]. Similarly, there was an increased loss of melanin concentrating hormone $(\mathrm{MCH})$ cells with disease severity. There are also some other neurotransmitters and proteins loss in PD: Hcrt; $\mathrm{MCH}$; alpha synuclein and glial fibrillary acidic protein cells were lost throughout the anterior to posterior extent of their hypothalamic distributions [34].

Adenosine and adenosine deaminize were observed with differences in PD and normal controls. Adenosine deaminize (ADA) and its iso-enzyme activities in serum were measured together with peripheral lymphocyte subsets in 42 patients with idiopathic Parkinson's disease. The total and ADA 2 activities were significantly higher than normal controls. As regards the peripheral lymphocyte subsets, the proportion of OKT $10+$ cells (activated $\mathrm{T}$ lymphocytes) and the proportions of interleukin-2 receptor + and HLA-DR + cells (mainly activated $\mathrm{T}$ lymphocytes) were significantly higher than normal controls. On the other hand, OKT $10+$ cells demonstrated a significant correlation not only with total ADA but also with ADA 2 activity [35].

ADA has variation in the brain and that maybe related to the individual pattern of the sleep-wake cycle (i.e., long and short sleepers). It has been demonstrated that adenosine agonists and antagonists strongly impact upon sleep [36, 37]. In addition, the level of adenosine varies across the sleep /wake cycle and increases following sleep deprivation. ADA is a key enzyme involved in the metabolism of adenosine. It was questioned, therefore, whether there are differences in ADA activity in brain regions relevant to sleep regulation.

The regional differences in ADA activity correlate with variations in the level of its mRNA [38, 39]. This suggests that spatial differences in ADA activity are the result of changes in the expression of the ADA gene. We postulate that ADA plays an important role in the mechanism that controls regional concentration of adenosine in the brain and thus, it is a part of the sleep-wake regulatory mechanism that could be individual related.

\section{ADENOSINE AND CAFFEINE IN PARKINSON DISEASE}

Sleep begins by the sleep-active cells in the hypothalamus. Adenosine and some pharmacological agents 
that induced sleep-like stages could be working in the same neuroanatomical regions that have been proposed as part of sleep induced mechanisms. The microinjection of triazolam into such sites [40, 41] induced sleep. Injections of triazolam, in the medial preoptic area [MPA) at the anterior hypothalamus, consistently enhanced sleep. This is an anatomically specific effect, while the injections into nearby structures (the lateral preoptic area, the horizontal limb of the diagonal band of Broca) had no effect on sleep. The basal forebrain and anterior hypothalamus have long been thought to have an important role in sleep regulation [42].

Neurons in that area also have a response to adenosine as well as benzodiazepines, and they are numerous in the preoptic anterior hypothalamus (POAH) (located medial to the cholinergic zone of the $\mathrm{BF}$ ) where they comprise about $25 \%$ of the cells recorded in vivo. Lesions of the POAH in rats and cats produce significant reductions in sleep. Microinjections of somnogens such as adenosine and prostaglandin D2 into the POAH significantly increase sleep [43].

Segregated groups of sleep-active neurons are localized in the ventrolateral preoptic area (VLPO) and median preoptic area (MnPO) [44]. These cells express Gamaamino-butyric acid (GABA), and in the VLPO neurons, GABA is co-localized with galanin. Data from c-fos studies as well as from electrophysiological and in vivo microdialysis studies suggest that VLPO neurons are active only during spontaneous sleep and recovery sleep, but not during periods of wakefulness preceding sleep, e.g., during $\mathrm{SD}$, suggesting that these neurons may not be involved in the mediation of the sleepiness associated with prolonged wakefulness, but rather in some aspect of maintenance or execution of sleep. However, the number of GABAergic neurons expressing c-Fos in the MnPO increase after $2 \mathrm{~h} \mathrm{SD}$ as compared with spontaneous sleep and recovery sleep [45].

Adenosine is the end product of hydrolysis of the universal "energy currency", ATP, thus energy consumption and adenosine formation are directly related. It is safe to postulate that energy metabolism is somehow involved in the regulation of sleep, since all normal brain functions require undisturbed energy metabolism. Several indirect lines of evidence suggest that energy metabolism decreases during sleep. Whether energy metabolism has a direct role in sleep regulation remains questionable, although many experiments support this notion. The so-called "homeostatic theory", stated that the depletion of brain glycogen stores during prolonged wakefulness leads to increase in sleep need [46]. However, recently this hypothesis has been modified. Lactate produced by astrocytes is the main source of energy to neurons, which introduces another variable that was not figured out before.

A more recent hypothesis suggests that neurons can use lactate as an energy source and even favors lactate over glucose. During times of activation the utilization of lactate as a source of energy for neuronal activation has been supported by a number of experiments. The core of this hypothesis is that upon neuronal activation, astrocytes metabolize glucose to lactate, which is then transported to neurons via selective transporters and that neurons use it as energy substrate instead of glucose. As the lactate that neurons utilize is produced by astrocytes and is transferred to neurons via the extracellular space, In Vivo micro dialysis measurement of lactate can be used as a marker of neuronal activation [47].

The extracellular concentration of adenosine increases in the cortex and basal forebrain during prolonged wakefulness and decreases during the sleep recovery period. Therefore, adenosine is proposed to act as a homeostatic regulator of sleep and to be a link between the humoral and neural mechanisms of sleep-wake regulation. Both the adenosine A [1] receptor $A_{1} R$ ) and $A_{2 A} R$ are involved in sleep induction. The $\mathrm{A}_{2 \mathrm{~A}} \mathrm{R}$ plays a predominant role in the somnogenic effects of PGD [2]. By use of gene-manipulated mice, the arousal effect of caffeine was shown to be dependent on the $A_{2 A} R$. On the other hand, inhibition of wake-promoting neurons via the $A_{1} R$ also mediates the sleep-inducing effects of adenosine, whereas activation of $A_{1} R$ in the lateral preoptic area induces wakefulness, suggesting that $A_{1} R$ regulates the sleep-wake cycle in a site-dependent manner $[48,49]$. Because these changes appear to be more pronounced in the basal forebrain (BF) than in other cerebral regions, local release of adenosine in $\mathrm{BF}$ was proposed to provide a signal for the homeostatic regulation of non-REM sleep [50].

Caffeine is a component of tea, coffee and cola drinks, and caffeine is the most commonly ingested methylxanthines. Moreover, caffeine, as well as related methylxanthines such as theophylline and aminophylline, is widely used as medications to treat asthma and apnea in the newborn. Caffeine is also present in many over-the-counter medications, such as in headache preparations in association with aspirin and in appetite suppressants [51].

About action mechanisms in the central nervous system there are at least three hypothesis: intracellular mobilization of calcium, inhibition of phosphodiesterases, and antagonism at the level of adenosine receptors [51].

Caffeine is the most frequently used central nervous system (CNS) stimulant in the world [52]. It is a methyl xanthine that is rapidly absorbed when taken orally, with a distribution volume similar to that of total body water and a half-life of approximately 3-8 h [53]. The waking effect of caffeine is likely due to a non-selective blockade of adenosine receptors, particularly the $\mathrm{A}_{1}$ and $\mathrm{A}_{2 \mathrm{~A}}$ receptors [48]. Antagonism of the $A_{1}$ adenosine receptors on wake active basal forebrain cholinergic neurons may prevent them from being silenced [54]. Similarly blockade of the adenosine $\mathrm{A} 2 \mathrm{~A}$ receptors may prevent the sleep-active neurons in the preoptic area from becoming active [55]. The effects of caffeine on sleep architecture have been shown in normal volunteers [48, 56] and insomniac patients [57], caffeine is capable of antagonizing the cognitive impairment produced by prolonged sleep deprivation [58]. In rats, we determined that a reduction in sensitivity or number of adenosine receptors might contribute to the decline in sleep drive with aging $[55,59]$.

\section{CAFFEINE INTAKE COULD HAVE A NEUROPROTECTIVE EFFECT IN PD}

In the "Honolulu Heart Program", the main finding was that caffeine intake has an inverse association with $\mathrm{PD}$, even after they correct the early study by tobacco smoking [60]. 
The finding was consistent whether coffee intake was determined by 24-hour recall or by food frequency questionnaire.

The pharmacological effects of caffeine could also modulate neurotransmitters and receptors systems of brainstem nuclei or striatum. Caffeine is a known central nervous system stimulant thought to act through adenosine receptor antagonism. Adenosine receptor agonists produce decreased locomotors activity in rodents, possibly through inhibition of dopamine neurotransmission [61].

Adenosine receptors modulate dopaminergic function by regulating dopamine release in presynaptic neurons and intracellular signaling in postsynaptic striatal neurons. To investigate how adenosine imposes on the action of dopamine in feeding and locomotion, genetically altered dopamine-deficient mice were treated with adenosine receptor antagonists. Acute administration of the nonselective adenosine receptor antagonist, caffeine [5-25 $\mathrm{mg} / \mathrm{kg}$ i.p.), reversed the hypophagia of mutant mice and induced hyperactivity in both control and mutant animals. However, caffeine treatment elicited much less hyperactivity in dopamine deficient mice than did L-3,4 dihydroxy phenylalanine (L-dopa) administration, which partially restores dopamine content [62].

Caffeine and tobacco had been postulated with some neuroprotective effects in PD. Inverse associations of smoking and caffeine were corroborated using families with PD, thus emphasizing smoking and caffeine as important covariates to consider in genetic studies of PD. The inverse association of smoking with PD was corroborated in twins and siblings. Meta-analysis suggests that the inverse association of PD with coffee drinking may be slightly weaker than the association with smoking [63]. To explore the possibility that caffeine helps prevent the dopaminergic deficits characteristic of PD, there was an investigation of the effects of caffeine and the adenosine receptor subtypes through which it may act in the 1-methyl-4-phenyl- 1,2,3,6tetrahydropyridine a [MPTP) neurotoxin model of PD.

Caffeine, at doses comparable to those of typical human exposure, attenuated MPTP-induced loss of striatal dopamine and dopamine transporter binding sites. These data establish a potential neural basis for the inverse association of caffeine with the development of $\mathrm{PD}$, and they enhance the potential of $\mathrm{A} 2 \mathrm{~A}$ antagonists as a novel treatment for this neurodegenerative disease [64] . The present data reveal a novel protective effect of caffeine on the pathophysiological responses of dopaminergic nigro striatal neurons in a mouse model of PD. Caffeine dose dependently attenuated the MPTP-induced depletion of functional and anatomical markers of the NS neurons targeted in PD. The neuroprotective actions of this nonspecific adenosine receptor antagonist were mimicked by multiple $\mathrm{A} 22_{\mathrm{A}}$-specific antagonists but not by an A1-specific antagonist. MPTP toxicity was also blunted in mice lacking the A2AR, confirming the role of this receptor in facilitating MPTP toxicity and supporting its contribution to the neuroprotective effect of caffeine [64].

Associations between PD and environmental measures (history, status, dosage, duration, and intensity) of smoking, coffee, caffeine, no steroidal anti-inflammatory drugs, and no steroidal anti-inflammatory drugs, were examined using generalized estimating equations with an independent correlation matrix while controlling for age and sex [65].

Individuals with PD were significantly less likely to report ever smoking. Additional measures of smoking revealed significant inverse associations with PD and trends in odds ratios. Increasing intensity of coffee drinking was inversely associated with PD. Increasing dosage and intensity of total caffeine drinking were also inversely associated, with high dosage presenting a significant inverse association for PD. There were no-significant associations between non-steroidal anti-inflammatory drugs and PD [65].

\section{HOW CAN BE PD PREDICTED USING SLEEP DISORDERS AS AN INFORMATION SOURCES}

Neuroprotection can be defined as the consequence of any intervention that produces enduring benefits by favorably influencing underlying etiology or pathogenesis and thereby forestalling onset of illness or clinical decline [14]. Neurorescue is defined as the capacity of any intervention to normalize the function of injured [but not dead) neurons [14]. This implies that neurons may be dysfunctional but not irreversibly damaged and therefore capable of being restored to normal functioning. Both concepts can be included under the umbrella of "disease modification', which can be seen as the clinical face of Neuroprotection and/or Neurorescue.

Idiopathic RBD has a very high predictive value, with approximately $50 \%$ of affected individuals developing PD or dementia within 10 years. This implies that idiopathic RBD patients are ideal candidates to test potential preclinical markers.

However, the specificity of symptom screens for RBD is not established, not all persons with PD develop RBD, and there are only some limited ways to predict which RBD patients will develop PD. Other simple screens based upon autonomic symptoms, depression and personality changes, quantitative motor testing and other sleep disorders may also be useful markers, but have not been extensively tested. Other more expensive measures such as detailed autonomic testing, cardiac scintillography, dopaminergic imaging and transcraneal ultrasound may be especially useful in defining disease risk in those identified through primary screening [66].

\section{IS CAFFEINE HYPERSENSITIVITY AND SLEEP PROBLEMS SOME KIND OF MARKER OF PD?}

Caffeine produces dose-dependent symptoms, and intoxication may develop with overconsumption [67-69].

Patients with caffeine intoxication have some clinical manifestation as nausea and emesis, which can be difficult to control. Additionally, less severe characteristic symptoms of high consumption of caffeine originate from the stimulatory and psychoactive properties of the drug. Patients may complain of agitation, nervousness, headache, tremor, and sleep disturbances $[70,71]$. More life-threatening manifestations that require additional testing and treatment include tacky-dysrhythmias and electrolyte disturbances, including hypokalemia, hypomagnesaemia, and hypophosphatemia. Hyperglycemia, metabolic acidosis with increased serum lactate, and seizures 
may also occur. Three mechanisms of action support caffeine action in the CNS: Intracellular mobilization of calcium, inhibition of phosphodiesterases, and antagonism at the level of adenosine receptors [57].

Anxiety disorder patients, specifically those with panic attacks are more prone to develop intolerance to caffeine.The following study compared patients with Panic Disorders (Panic D), Major Depression (MD) and MD with Panic D (MDP). The dose of 480-mg of oral caffeine challenge test was performed with a control group. The result indicates that those subjects who have anxiety problems are more likely to panic in response to the caffeine challenge test [72].

Some people that are sensitive to the anxiogenic effects of caffeine could be because some polymorphisms in the genes that encodes receptors where this drug acts. Thus, functional polymorphisms in genes relating to the central actions of caffeine may be related to anxiogenic responses.

That has been explored before [73]. In such studies it was observed an association between self-reported anxiety after caffeine and a single-nucleotide polymorphism (SNP) in the gene encoding the adenosine $\mathrm{A} 2_{\mathrm{A}}$ receptor $\left(\mathrm{ADORA} \mathrm{A}_{2 \mathrm{~A}}\right)$.

\section{ADENOSINE AND DOPAMINE RECEPTORS}

The relationship between adenosine and dopamine receptors could be summarized as follows: antagonizing the actions of adenosine by caffeine indirectly potentiates dopaminergic signaling. Indeed, dopaminergic manipulations affect the behavioral properties of caffeine.Adenosine

$A_{1}$ and $A_{2 A}$ receptors form functional heteromeric complexes with dopamine $\mathrm{D}_{1}$ and $\mathrm{D}_{2}$ receptors [74] and there is evidence that these receptors interact at multiple levels to control cell function. First, the receptors physically interact and binding of adenosine to $\mathrm{A}_{2 \mathrm{~A}}$ reduces affinity of $\mathrm{DRD}_{2}$ for dopamine $[75,76]$. Second, there is reciprocal antagonism at the second messenger level reducing G-protein coupling and signaling. Activation of the $\mathrm{A}_{2 \mathrm{~A}}$ receptor stimulates adenylyl cyclase activity whereas activation of $\mathrm{DRD}_{2}$ inhibits adenylyl cyclase activity. Thus activation of $\mathrm{A} 2_{\mathrm{A}}$ uncouples DRD2 from its $G$ protein and reduces DRD2 signaling. Conversely, activation of DRD2 counteracts signaling via $\mathrm{A}_{2 \mathrm{~A}}$ [77].

The motor stimulant effects of caffeine are reduced in DRD2 knockout mice [64]. The anxiogenic induction of caffeine in healthy male and female individuals were studied. Subject who consumed less than $300 \mathrm{mg}$ caffeine per week, ingested capsules containing $0,50,150$, and $450 \mathrm{mg}$ caffeine under double-blind conditions in four separate experimental sessions. Subjective anxiety was measured before and at repeated times after capsules were consumed. At the $150 \mathrm{mg}$ dose of caffeine, it was found a significant association between caffeine-induced anxiety (Visual Analog Scales, VAS) and ADORA2Ars5751876 (1976C/T), rs2298383 (intron 1a) and rs4822492 (30-flank), and DRD2 rs1110976 (intron 6). Caffeine-induced anxiety (VAS) was also associated with two-loci interactions of selected ADORA2A and DRD2 polymorphisms. The lowest dose of caffeine did not increase ratings of anxiety while the highest dose increased anxiety in the majority of subjects. These findings provide support for an association between an ADORA2A polymorphism and self-reported anxiety after a moderate dose of caffeine. It is likely that other ADORA2A and DRD2 polymorphisms also contribute to responses to caffeine [78].

In support of the previous findings, there are some evidences from studies with old rats. There are significant decrements in sleep with age. These include fragmentation of sleep, increased wake time, decrease in the length of sleep bouts, decrease in the amplitude of the diurnal rhythm of sleep, decrease in rapid eye movement sleep and a profound decrease in electroencephalogram $\Delta$ power $(0.3-4 \mathrm{~Hz}]$. Old rats also have less sleep in response to $12 \mathrm{~h}$ of prolonged wakefulness (W). That could indicate a reduction in sleep drive as something related to the old age. The mechanism contributing to the decline in sleep with aging is not known but cannot be attributed to loss of neurons implicated in sleep since the numbers of neurons in the ventral lateral preoptic area, a region implicated in generating sleep, is similar between young ( 3.5 months) and old (21.5 months) rats. One possibility for the reduced sleep drive with age is that sleep-wake active neurons may be stimulated less as a result of a decline in endogenous sleep factors. Adenosine $(\mathrm{AD})$, is one of such sleep factor that increases after prolonged wakefulness (W). In experiment 1 , microdialysis measurements of $\mathrm{AD}$ in the basal forebrain at $1 \mathrm{~h}$ intervals reveal that old (21.5 months) rats have more extracellular levels of AD compared with young rats across the $24 \mathrm{~h}$ diurnal cycle. In experiment 2, old rats kept awake for $6 \mathrm{~h}$ (first half of lights-on period) accumulated more AD compared with young rats. If old rats have more $\mathrm{AD}$ then why do they sleep less? To investigate whether changes in sensitivity of the $\mathrm{AD}$ receptor contribute to the decline in sleep, experiments 3 and 4 determined that for the same concentration of $\mathrm{AD}$ or the $\mathrm{AD}$ receptor 1 agonist, cyclohexyladenosine, old rats have less sleep compared with young rats. It was concluded that even though old rats have more $\mathrm{AD}$, a reduction in the sensitivity of the $\mathrm{AD}$ receptor to the ligand does not transduce the $\mathrm{AD}$ signal at the same strength as in young rats and may be a contributing factor to the decline in sleep drive in the elderly [54].

\section{CONCLUSION}

The relationship between Parkinson disease, sleep, adenosine and caffeine, has been very useful to understand basic processes of sleep physiology and motor control, as well as neurological pathology. But at the same time, they can be the tools to detect the early beginnings of neurodegenerative diseases such PD. We must develop early detection programs, with periodic evaluations of factors that may go unnoticed to the patients and their families. Sleep monitorization with home devices, coupled with home computers. That could be available for home use in patients with some sleep disorders that start after the third or fourth decade of life, together with anosmia, constipation and major depression or anxiety disorders. That would be relevant on people with disproportionate caffeine side effects manifestation.

It was confirmed that patients with PD have a low consumption of caffeine from an early age. There is now evidence, that is happens because some people may be a great sensitivity for the stimulant effects of caffeine. One goal in the future would be to explore in people in the fourth decade, the tolerance of low doses of caffeine, because they could be at some risk to develop PD. Of course that with 
some other early non-motor markers as sleep disorders, anosmia, intestinal problems and psychiatric disorders. We can slow down the neurodegeneration and allow people to be less incapacitated than other patients before. That is a short step, but for sure it is worthy.

\section{ABBREVIATIONS}

\begin{tabular}{|c|c|c|}
\hline $\mathrm{AD}$ & $=$ & Adenosine \\
\hline ADA & $=$ & Adenosine Deaminize. \\
\hline ADORA2A & $=$ & Adenosine $\mathrm{A} 2 \mathrm{~A}$ receptor. \\
\hline ATP & $=$ & Adenosine Triphophate. \\
\hline CNS & $=$ & Central Nervous System. \\
\hline DA & $=$ & Dopamine. \\
\hline $\mathrm{BF}$ & $=$ & Basal Forebrain. \\
\hline DBS & $=$ & Deep Brain Stimulation. \\
\hline GABA & $=$ & Gama-amino-butyric acid. \\
\hline Hert & $=$ & Hypocretin. \\
\hline $\mathrm{MCH}$ & $=$ & Melanin concentrating hormone. \\
\hline $\mathrm{MD}$ & $=$ & Major Depression. \\
\hline MDP & $=$ & MD with PD. \\
\hline $\mathrm{MnPO}$ & $=$ & Medial Preoptic Area \\
\hline MPTP & $=$ & $\begin{array}{l}\text { 1-methyl-4-phenyl- } \\
\text { dropyridine. }\end{array}$ \\
\hline OKT & $=$ & $\begin{array}{l}\text { Ortho-Kung } \mathrm{T} \text { cells. Thosecells } \\
\text { recognized by monoclonal antibod } \\
\mathrm{T} \text {-lymphocyte antigens. }\end{array}$ \\
\hline Panic D & $=$ & Panic Disorders. \\
\hline PD & $=$ & Parkinson Disease. \\
\hline PG & $=$ & Prostaglandins. \\
\hline POAH & $=$ & Preoptic Anterior Hypothalamus. \\
\hline RBD & $=$ & REM Sleep Behavior Disorder. \\
\hline REM & $=$ & Rapid Eye Movement. \\
\hline $\mathrm{SN}$ & $=$ & Substantia Nigra. \\
\hline SNpc & $=$ & Substantia Nigra pars compacta \\
\hline SNP & $=$ & Single-nucleotide polymorphism. \\
\hline VAS & $=$ & Visual Analog Scales \\
\hline VLPO & $=$ & Ventral Lateral Pre Optic \\
\hline
\end{tabular}

\section{CONFLICT OF INTEREST}

The authors confirm that this article content has no conflicts of interest.

\section{ACKNOWLEDGEMENTS}

No financial contribution has support this work, other than my University (National Autonomous University of Mexico).

\section{REFERENCES}

[1] Roth T. Conclusion: directions for future research into optimizing outcomes measurement in sleep medicine. Sleep Med 2008; 9 (Suppl 1): S35.

[2] Roth T. Sleep disorders medicine. Henry Ford Hospital Med J 1988; 36: 4-10.
[3] Kryger MH, Roth T, Dement WC. Principles and practice of sleep medicine Basic Edition. $5^{\text {th }}$ ed. St. Louis, Missouri USA: Elsevier Saunders 2011.

[4] Heisters D. Parkinson's: symptoms, treatments and research. Br J Nurs 2011; 20: 548-54.

[5] Sharma A, Szeto K, Desilets AR. Efficacy and safety of deep brain stimulation as an adjunct to pharmacotherapy for the treatment of Parkinson disease. Ann Pharmacother 2012; 46(2): 248-54.

[6] Harries AM, Kausar J, Roberts SA, et al. Deep brain stimulation of the subthalamic nucleus for advanced Parkinson disease using general anesthesia: long-term results. J Neurosurg 2012; 116: 107-13.

[7] Jecmenica-Lukic M, Poewe W, Tolosa E, Wenning GK. Premotor signs and symptoms of multiple system atrophy. Lancet Neurol 2012; 11:361-8.

[8] Merola A, Zibetti M, Angrisano S, et al. Parkinson's disease progression at 30 years: a study of subthalamic deep brain-stimulated patients. Brain 2011; 134: 2074-84.

[9] Weerkamp NJ, Nijhof A, Tissingh G. [Non-motor symptoms of Parkinson's disease]. Nederlands tijdschrift voor geneeskunde. 2012; 156: A3926.

[10] Postuma RB, Aarsland D, Barone P, et al. Identifying prodromal Parkinson's disease: pre-motor disorders in Parkinson's disease. Mov Dis 2012; 27: 617-26.

[11] Martinez-Martin P. The importance of non-motor disturbances to quality of life in Parkinson's disease. J Neurol Sci 2011; 310(1-2): 1216.

[12] Savica R, Rocca WA, Ahlskog JE. When does parkinson disease start? Arch Neurol 2010; 67: 798-801.

[13] Wu Y, Le W, Jankovic J. Preclinical biomarkers of Parkinson disease. Arch Neurol 2011; 68: 22-30.

[14] Shapira A, Olanow C. Neuroprotection in Parkinson's disease: mysteries, myths and misconceptions. JAMA 2004; 291: 8-12.

[15] Wichmann T, DeLong M. Functional and pathophysiological models of the basal ganglia. . Curr Opin Neurobiol 1996; 6: 7-11.

[16] Quartarone A, Siebner H, Rothwell J. Task-specific hand dystonia: can too much plasticity be bad for you? Trends Neurosci 2006; 29: 7-9.

[17] Cooke S, Bliss TV. Plasticity in the human central nervous system. Brain 2006; 129: 1659-73.

[18] Bezard E, Gross CE, Brotchie JM. Presymptomatic compensation in Parkinson's disease is not dopamine-mediated. Trends Neurosci 2003; 26: 6-10.

[19] Miyamoto T. Olfactory dysfunction in parkinson disease and rem sleep behavior disorder. Brain Nerve 2012; 64: 356-63.

[20] Ashraf W, Pfeiffer RF, Park F, et al. Constipation in Parkinson's disease: objective assessment and response to psyllium. Mov Disord 1997; 12: 946-51.

[21] Aarsland D, Bronnick K, Alves G, et al. The spectrum of neuropsychiatric symptoms in patients with early untreated Parkinson's disease. J Neurol Neurosurg Psychiatr 2009; 80: 928-30.

[22] Ishihara L, Brayne C. What is the evidence for a premorbid parkinsonian personality: a systematic review. Mov Disord 2006; 21: 7-15.

[23] Lai YY, Siegel JM. Physiological and anatomical link between Parkinson-like disease and REM sleep behavior disorder. Mol Neurobiol 2003; 27: 137-52.

[24] Poryazova R, Waldvogel D, Bassetti CL. Sleepwalking in Patients With Parkinson Disease. Arch Neurol 2007; 64: 1524-7.

[25] Grinberg LT, Rueb U, Alho AT, Heinsen H. Brainstem pathology and non-motor symptoms in PD. J Neurol Sci 2010; 289: 81-8.

[26] Hasegawa K. Does early detection of non-motor symptoms facilitate early treatment of Parkinson's disease? Brain Nerve 2012; 64: 453-61.

[27] Postuma RB, Gagnon JF, Vendette M, Montplaisir JY. Markers of neurodegeneration in idiopathic rapid eye movement sleep behaviour disorder and Parkinson's disease. Brain 2009; 132: 3298-307.

[28] Romenets SR, Wolfson C, Galatas C, et al. Validation of the nonmotor symptoms questionnaire (NMS-Quest). Parkinson Relat Dis 2012; 18: 54-8.

[29] Boot BP, Boeve BF, Roberts RO, et al. Probable rapid eye movement sleep behavior disorder increases risk for mild cognitive impairment and Parkinson disease: a population-based study. Ann Neurol 2012; 71: 49-56.

[30] Louter $\mathrm{M}$, Aarden WC, Lion J, Bloem BR, Overeem $\mathrm{S}$. Recognition and diagnosis of sleep disorders in Parkinson's disease. J Neurol 2012. (In press) 
[31] Chen H, Schernhammer E, Schwarzschild MA, Ascherio A. A prospective study of night shift work, sleep duration, and risk of parkinson's disease. Am J Epidemiol 2006; 163: 726-30.

[32] De Cock VC, Vidailhet $\mathrm{M}$, Leu S, et al. Restoration of normal motor control in Parkinson's disease during REM sleep. Brain 2007; 130: 450-6.

[33] Fronczek R, Overeem S, Lee SYY, et al. Hypocretin (orexin) loss in Parkinson's disease. Brain 2007; 130: 1577-85.

[34] Thannickal TC, Lai YY, Siegel JM. Hypocretin (orexin) cell loss in Parkinson's disease. Brain J Neurol 2007; 130(6): 1586-95.

[35] Mackiewicz M, Nikonova EV, Zimmerman JE, et al. Enzymes of adenosine metabolism in the brain: diurnal rhythm and the effect of sleep deprivation. J Neurochem 2003; 85: 348-57.

[36] Satoh S, Matsumura H, Hayaishi O. Involvement of adenosine A2A receptor in sleep promotion. Eur J Pharmacol 1998; 351: 155-62.

[37] Satoh S, Matsumura H, Suzuki F, Hayaishi O. Promotion of sleep mediated by the A2a-adenosine receptor and possible involvement of this receptor in the sleep induced by prostaglandin D2 in rats. Proc Natl Acad Sci 1996; 93: 5980-4.

[38] Bjorness TE, Greene RW. Adenosine and sleep. Curr Neuropharmacol 2009; 7: 238-45.

[39] Bjorness TE, Kelly CL, Gao T, Poffenberger V, Greene RW. Control and function of the homeostatic sleep response by adenosine A1 receptors. J Neurosci 2009; 29: 1267-76.

[40] Mendelson WB. Neuropharmacology of sleep induction by benzodiazepines. Crit Rev Neurobiol 1992; 6: 221-32.

[41] Mendelson WB, Martin JV. Characterization of the hypnotic effects of triazolam microinjections into the medial preoptic area. Life Sci 1992; 50: 1117-28.

[42] Mendelson WB. Sleep-inducing effects of adenosine microinjections into the medial preoptic area are blocked by flumazenil. Brain Res 2000; 852: 479-81.

[43] Porkka-Heiskanen T, Kalinchuk AV. Adenosine, energy metabolism and sleep homeostasis. Sleep Med Rev 2011; 15: 123-35.

[44] Szymusiak R, McGinty D. Hypothalamic regulation of sleep and arousal. Ann Acad NY Sci 2008; 1129: 275-86.

[45] Gvilia I, McGinty D, Szymusiak R. Homeostatic regulation of sleep: a role for preoptic area neurons. . J Neurosci 2006; 26: 5-10.

[46] Benington JH, Heller HC. Restoration of brain energy metabolism as the function of sleep. Prog Neurobiol 1995; 45: 347-60.

[47] Fillenz M. The role of lactate in brain metabolism. Neurochem Int 2005; 47: 4-12.

[48] Huang ZL, Urade Y, Hayaishi O. The role of adenosine in the regulation of sleep. Curr Top Med Chem 2011; 11: 1047-57.

[49] Diaz-Munoz M, Salin-Pascual R. Purine molecules as hypnogenic factors role of adenosine, ATP, and caffeine. Central Nerv Syst Agents Med Chem 2010; 10: 259-68.

[50] Basheer R, Strecker RE, Thakkar MM, McCarley RW. Adenosine and sleep-wake regulation. Prog Neurobiol 2004; 73: 379-96.

[51] Nehlig A, Daval J-L, Debry GC. Caffeine and the central nervous system: mechanisms of action,biochemical, metabolic and psychostimulant effects. Brain Res Rev 1992; 17: 31-40.

[52] Sawyer DA, Julia HL, Turin AC. Caffeine and human behavior: arousal, anxiety, and performance effects. J Behav Med 1982; 5: 415-439.

[53] Yang A, Palmer AA, de Wit H. Genetics of caffeine consumption and responses to caffeine. Psychopharmacol 2010; 211:245-57.

[54] Blanco-Centurion C, Xu M, Murillo-Rodriguez E, et al. Adenosine and sleep homeostasis in the Basal forebrain. J Neurosci 2006; 26: 8092-100

[55] Murillo-Rodriguez E, Blanco-Centurion C, Gerashchenko D, SalinPascual RJ, Shiromani PJ. The diurnal rhythm of adenosine levels in the basal forebrain of young and old rats. Neurosci 2004; 123: 361-70.

[56] Landolt HP, Roth C, Dijk DJ, Borbely AA. Late-afternoon ethanol intake affects nocturnal sleep and the sleep EEG in middle-aged men. J Clin Psychopharmacol 1996; 16: 428-36.

[57] Salin-Pascual RJ, Valencia-Flores M, Campos RM, Castano A, Shiromani PJ. Caffeine challenge in insomniac patients after total sleep deprivation. Sleep Med 2006; 7: 141-5.
[58] Porkka-Heiskanen T. Adenosine in sleep and wakefulness. Ann Med 1999; 31: 125-9.

[59] Salin-Pascual RJ, Upadhyay U, Shiromani PJ. Effects of hypocaloric diet on sleep in young and old rats. Neurobiol Aging 2002; 23: 771-6.

[60] Ross GW, Abbott RD, Petrovitch H, et al. Association of Coffee and Caffeine Intake With the Risk of Parkinson Disease. JAMA: 2000; 283: 2674-9.

[61] Jones FS, Jing J, Stonehouse AH, Stevens A, Edelman GM. Caffeine stimulates cytochrome oxidase expression and activity in the striatum in a sexually dimorphic manner. Mol Pharmacol 2008; 74: 673-84.

[62] Kim DS, Palmiter RD. Adenosine receptor blockade reverses hypophagia and enhances locomotor activity of dopamine-deficient mice. Proc Natl Acad Sci 2003; 100: 1346-51.

[63] Hernan M, Takkouche B, Caamano-Isorna F, Gestal-Otero J. A meta-analysis of coffee drinking, cigarette smoking, and the risk of Parkinson's disease. Ann Neurol 2002; 52: 8-14.

[64] Chen J-F, Xu K, Petzer JP, et al. Neuroprotection by caffeine and a2a adenosine receptor inactivation in a model of Parkinson's disease. J Neurosci 2001; 21: RC143.

[65] Hancock DB, Martin ER, Stajich JM, et al. Smoking, caffeine, and nonsteroidal anti-inflammatory drugs in families with Parkinson disease. Arch Neurol 2007; 64: 576-80.

[66] Postuma RB, Gagnon JF, Montplaisir J. Clinical prediction of Parkinson's disease: planning for the age of neuroprotection. J Neurol Neurosurg Psychiatr 2010; 81: 1008-13.

[67] Luciano M, Kirk KM, Heath AC, Martin NG. The genetics of tea and coffee drinking and preference for source of caffeine in a large community sample of Australian twins. Addiction 2005; 100: 1510-7.

[68] Stafford LD, Yeomans MR. Caffeine deprivation state modulates coffee consumption but not attentional bias for caffeine-related stimuli. Behav Pharmacol 2005; 16: 559-71.

[69] Svikis DS, Berger N, Haug NA, Griffiths RR. Caffeine dependence in combination with a family history of alcoholism as a predictor of continued use of caffeine during pregnancy. Am J Psychiatr 2005; 162: 2344-51.

[70] Fernandez-Miret B, Castano Avila S, Maynar Moliner J, Iturbe Rementeria M, Barrasa Gonzalez H, Corral Lozano E. Severe acute caffeine intoxication. A report of two cases with different outcomes. Medicina intensiva / Sociedad Espanola de Medicina Intensiva y Unidades Coronarias 2012 (In press).

[71] Wolk BJ, Ganetsky M, Babu KM. Toxicity of energy drinks. Curr Open Pediatr 2012; 24: 243-51.

[72] Nardi AE, Lopes FL, Freire RC, et al. Panic disorder and social anxiety disorder subtypes in a caffeine challenge test. Psychiatr Res 2009; 169: 149-53.

[73] Alsene K, Deckert J, Sand P, de Wit H. Association between A2a receptor gene polymorphisms and caffeine-induced anxiety. Neuropsychopharmacol 2003; 28: 1694-702.

[74] Fuxe K, Agnati LF, Jacobsen K, et al. Receptor heteromerization in adenosine A2A receptor signaling: relevance for striatal function and Parkinson's disease. Neurol 2003; 6: S19-23.

[75] Torvinen M, Marcellino D, Canals M, et al. Adenosine A2A receptor and dopamine D3 receptor interactions: evidence of functional A2A/D3 heteromeric complexes. Mol Pharmacol 2005; 67: 400-407.

[76] Torvinen M, Torri C, Tombesi A, et al. Trafficking of adenosine A2A and dopamine D2 receptors. J Mol Neurosci 2005; 25: 191-200.

[77] Fuxe K, Ferre S, Canals M, et al. Adenosine A2A and dopamine D2 heteromeric receptor complexes and their function. J Mol Neurosci 2005; 26: 209-20.

[78] Childs E, Hohoff C, Deckert J, Xu K, Badner J, de Wit H. Association between ADORA2A and DRD2 polymorphisms and caffeine-induced anxiety. Neuropsychopharmacol 2008; 33: 2791800 .

(c) Rafael J. Salin-pascual; Licensee Bentham Open.

This is an open access article licensed under the terms of the Creative Commons Attribution Non-Commercial License (http://creativecommons.org/licenses/by$\mathrm{nc} / 3.0 /$ ), which permits unrestricted, non-commercial use, distribution and reproduction in any medium, provided the work is properly cited 